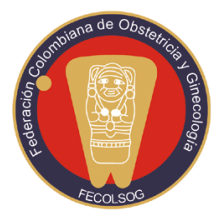

Revista Colombiana de Obstetricia y Ginecología Vol. 70 No. $3 \bullet J u l i o-S e p t i e m b r e ~ 2019 \bullet(165-173$ )

INVESTIGACIÓN ORIGINAL

DOI: https://doi.org/10.18597/roog.3177

\title{
SEGURIDAD Y EFICACIA DE LA VAGINOPLASTIA DE VECCHIETTI EN AGENESIA VAGINAL: ESTUDIO DE COHORTE, EN MEDELLÍN, COLOMBIA, 2007 A 2012
}

\section{Safety and efficacy of Vecchietti vaginoplasty in vaginal agenesis: colhort study. Medellín, Colombia 2007-2012}

\section{Segurança e eficácia dla vaginoplastia de Vecchietti} na agenesia vaginal: coorte de 2007 a 2012

Jorge Enrique Aristizábal-Duque, MD, $M_{S c^{1 *}}$; Joan Camilo Piedrahíta-Mejía, $M D, M S C^{2}$

Recibido: junio 14/18 - Aceptado: septiembre 16/19

\section{RESUMEN}

Objetivo: describir la técnica de vaginoplastia de Vecchietti (TVV) en pacientes diagnosticadas con agenesia vaginal secundaria y hacer una aproximación a la seguridad y eficacia de esta técnica.

Materiales y métodos: cohorte histórica de pacientes con agenesia vaginal secundaria al síndrome de Mayer-Rokitansky-Kuster-Hauser y al síndrome de insensibilidad androgénica, a quienes se les realizó vaginoplastia por técnica de Vecchietti en el Hospital Universitario San Vicente Fundación, institución de referencia, de alta complejidad, en el periodo 2007 a 2012. Se excluyeron quienes te-

* Correspondencia: Jorge Enrique Aristizábal Duque (investigador principal), carrera $51 \mathrm{D} \mathrm{N}^{\circ}$ 62-69/HUSVF, bloque 12 (maternidad). Medellín, Colombia. jead1@une.net.co

Médico y cirujano; especialista en Ginecología y Obstetricia; especialista en Medicina Reproductiva. Profesor del Departamento de Ginecología y Obstetricia de la Universidad de Antioquia, Medellín, Colombia. jead1@une.net.co

2 Médico y cirujano; especialista en Epidemiología; especialista en Edición de Publicaciones. Profesor del Departamento de Ginecología y Obstetricia de la Universidad de Antioquia, Medellín, Colombia. nían una vagina funcional para relaciones coitales. Muestreo consecutivo. Se midieron variables sociodemográficas, clínicas, de seguridad y de eficacia. Se utilizó estadística descriptiva.

Resultados: el principal motivo de consulta fue la amenorrea primaria (69,2\%). Las malformaciones asociadas fueron agenesia renal derecha $(15,4 \%)$ y malformaciones esqueléticas $(15,4 \%)$. Se presentó una perforación intraoperatoria de la vejiga y tres complicaciones menores $(23,1 \%)$ en el posoperatorio. En el 84,6\% de ellas se obtuvo una vagina funcional a un año de seguimiento.

Conclusiones: la TVV es una técnica quirúrgica simple que ha permitido obtener resultados funcionales satisfactorios con complicaciones menores. Se requieren estudios con grupo control para tener una mejor evaluación de la eficacia de las diferentes técnicas de construcción de la neovagina.

Palabras clave: procedimientos quirúrgicos reconstructivos; enfermedades vaginales; síndrome de resistencia androgénica. 


\section{ABSTRACT}

Objective: To describe the Vecchietti vaginoplasty technique (VVT) in patients diagnosed with secondary vaginal agenesis and to analyze the safety and efficacy of this technique.

Materials and methods: Historical cohort of patients with vaginal agenesis secondary to MayerRokitansky-Kuster-Hauser and androgen insensitivity syndromes, subjected to vaginoplasty using the Vecchietti technique at San Vicente Fundación University Hospital, a high complexity referral institution located in the city of Medellín, during the time period between 2007 and 2012. Patients with functional a vagina for intercourse were excluded. Sampling was consecutive. Sociodemographic, clinical, safety and efficacy variables were measured. Descriptive statistics were used.

Results: The main complaint was primary amenorrhea (69.2\%). Associated malformations included right renal agenesis (15.4\%) and skeletal malformations (15.4\%). There was one intra-operative bladder perforation and, postoperatively, there were three (23.1\%) minor complications. At 1-year follow-up, a functional vagina had been obtained in $84.6 \%$ of cases.

Conclusion: Vecchietti vaginoplasty is a simple surgical technique resulting in satisfactory functional outcomes with only minor complications. Further studies with control groups are required in order to better assess the efficacy of the various techniques used for neovagina creation.

Key words: reconstructive surgical procedures; vaginal diseases; androgen resistance syndrome.

\section{RESUMO}

Objetivo: descrever a técnica da vaginoplastia de Vecchietti (TVV) em pacientes diagnosticadas com agenesia vaginal secundária e fazer uma abordagem a respeito da segurança e eficácia desta técnica.

Materiais e métodos: coorte histórica de pacientes com agenesia vaginal secundária à síndrome de Mayer-Rokitansky-Kuster-Hauser e à síndrome de insensibilidade androgênica, que foram submetidas a uma vaginoplastia pela técnica de Vecchietti no Hospital Universitário San Vicente Fundación, estabelecimento de referência, de alta complexidade, no período 2007-2012. Foram excluídas aquelas pacientes que tinham uma vagina funcional para relações coitais. Amostragem consecutiva. Foram mensuradas diversas variáveis sociodemográficas, clínicas, de segurança e de eficácia, utilizando o método de estatística descritiva.

Resultados: o principal motivo de consulta foi a amenorreia primária $(69,2 \%)$. As malformações correlatas foram agenesia renal direita $(15,4 \%)$ e malformações esqueléticas (15,4\%). Verificou-se uma perfuração intraoperatória da bexiga e três complicações menores $(23,1 \%)$ no pós-operatório. Em 84,6\% delas conseguiu-se uma vagina funcional durante o primeiro ano de acompanhamento.

Conclusão: a TVV é uma técnica cirúrgica simples que permitiu obter resultados funcionais satisfatórios com complicações menores. É preciso levantar estudos com grupo-controle para fazer uma melhor avaliação da eficácia das diferentes técnicas de construção da neovagina.

Palavras chave: procedimentos cirúrgicos reconstrutivos; doenças vaginais; síndrome de resistência androgênica.

\section{INTRODUCCIÓN}

La agenesia vaginal es la ausencia congénita parcial o completa de la vagina, causada por dos trastornos principales: el síndrome de agenesia útero-vaginal o de Mayer-Rokitansky-Kuster-Hauser (MRKH), cuya prevalencia es de 1/5.000 nacidas vivas, y el síndrome de insensibilidad androgénica (AIS, por su sigla en inglés), con una prevalencia estimada de 1/50.000 nacidas vivas. En todos los casos hay ausencia o alteración estructural del útero, por lo cual siempre afecta las funciones sexual y reproductiva (1).

Se han diseñado diferentes estrategias para crear una vagina, las cuales pueden ser agrupadas en técnicas quirúrgicas y métodos de dilatación mecánica. Las técnicas quirúrgicas pueden ofrecer un 
resultado definitivo inmediato o requerir del uso de algún tipo de molde vaginal por un tiempo variable, luego de la cirugía. Entre ellas están: los diferentes colgajos intestinales, la técnica de Williams con sus variantes, la técnica de Davydov y el uso de colgajos cutáneos de espesor total (2). La técnica de Williams permite obtener un saco vestibular por medio de una vulvoplastia, sin disección del espacio rectovesical. No requiere del uso de dilatadores, sin embargo, tiene limitaciones anatómicas (3). Las técnicas de colgajos ileal y rectosigmoidal son las favoritas de muchos cirujanos, pero también son las más complejas y las que presentan mayor frecuencia de complicaciones graves (4). En el abordaje de Davydov, se recubre el espacio rectovesical ampliado con peritoneo, a través de una técnica laparoscópica (5).

En cuanto a la dilatación manual progresiva es una estrategia simple, pero requiere de más tiempo, disponibilidad de personal de apoyo entrenado y compromiso por parte de la paciente para el uso diario del dilatador (6). En esta alternativa se encuentran las técnicas de McIndoe y sus variantes, la de Shears y la de Vechietti (7). La técnica de Shears utiliza como estrategia la dilatación quirúrgica progresiva de los dos canales müllerianos que desembocan en la placa genital, a través del espacio rectovesical. No requiere recubrimiento tisular del espacio obtenido, pero sí del uso de dilatadores por tiempo variable (8). La técnica de McIndoe es también una modalidad compleja, en la cual se introduce un molde sintético recubierto de un colgajo de piel libre del muslo o del área glútea en el espacio rectovesical (9).

En 1979, Vecchietti publicó la experiencia acumulada durante 14 años de una técnica de dilatación por un medio quirúrgico relativamente simple en 307 casos de agenesia vaginal de diferente origen (10). En 1992, Gauwerky introdujo el abordaje laparoscópico (11). Desde entonces, el volumen de la experiencia y los excelentes resultados a largo plazo han hecho que esta técnica tenga una amplia aceptación en Europa (12). En nuestro caso, el proceso de conocimiento de la técnica y el instrumental apropiado, el cual es semejante al original, se obtuvo gracias a la colaboración de los profesores Bernard Hedon y François Laffargue, del servicio de Ginecología y Obstetricia del Hospital Arnaud de Villeneuve en Montpellier, adscrito a la Universidad de Montpellier I, Francia.

Esta técnica es poco utilizada en Colombia y en la región latinoamericana, razón por la cual no se encuentra literatura respecto de las complicaciones de la misma o su eficacia, por lo que el objetivo de este estudio es describir la técnica de vaginoplastia de Vecchietti (TVV) en pacientes con agenesia vaginal secundaria a MRKH y AIS, y hacer una aproximación a la eficacia y a la seguridad del procedimiento.

\section{MATERIALES Y MÉTODOS}

Diseño y población. Estudio de cohorte histórica. Se incluyeron mujeres con diagnóstico de MKRH y AIS, a quienes se les practicó TVV entre los años 2007 y 2012, en el Hospital Universitario San Vicente Fundación, institución de referencia, de alta complejidad, ubicada en la ciudad de Medellín, en la región centro-occidental de Colombia. Se excluyeron quienes tenían una vagina funcional para relaciones coitales. Esta institución atiende pacientes del régimen contributivo en el sistema de seguridad social en Colombia. Se hizo muestreo consecutivo.

Procedimiento. A partir del registro de los casos intervenidos en la institución desde que se instauró la técnica en el año 2007 se identificaron las mujeres intervenidas por esta técnica. El investigador principal obtuvo la información directamente de la historia clínica electrónica de la institución (SAP), por medio de un formulario especialmente diseñado para el estudio.

Estudios preoperatorios: en las pacientes con MKRH se descartaron malformaciones renales y vertebrales por medio de ultrasonido o resonancia magnética. Los casos de AIS requirieron de imágenes pélvicas para aclarar la condición de las gónadas y una medición de testosterona total. No 
se solicitó cariotipo cuando se disponía de estudio anatomopatológico de las gónadas. Todas fueron intervenidas con un procedimiento estandarizado que realizó el mismo cirujano principal.

Descripción de la técnica quirúrgica: las pacientes se hospitalizaron el mismo día del procedimiento e ingresaron a sala de cirugía. Bajo anestesia se realizó lavado, asepsia y antisepsia de la región perineal, y en posición ginecológica se practicó laparoscopia convencional con el puerto umbilical de $11 \mathrm{~mm}$ y los puertos pélvicos laterales de $6 \mathrm{~mm}$. Se continuó con el abordaje perineal. Con el dedo índice de la mano no dominante en el canal rectal, se introduce una aguja con mandril 14 gauge en el espacio recto-vesical hacia el abdomen. Por medio de la laparoscopia se verifica que la aguja salga justo en el cordón fibroso que reemplaza al útero, sin comprometer la vejiga o el recto. $\mathrm{Al}$ aparecer la aguja en la cavidad abdominal se fija con una pinza fuerte provista de cerrojo, introducida por cualquiera de los puertos laparoscópicos pélvicos (figura 1).

Se practica luego una cistoscopia para descartar la perforación de la vejiga. Si se demuestra perforación de la vejiga se retira la aguja y se repite el procedimiento hasta confirmar la posición correcta. Posteriormente, se extrae el mandril de la aguja y se pasan a través de ella dos hilos de polyglactin calibre 1 hasta la cavidad abdominal. Luego se reparan con una pinza Maryland y, en este momento, se retira la aguja. En el extremo perineal se anuda una esfera de teflón de alta densidad de $22 \mathrm{~mm}$ de diámetro a los dos hilos, cuidando de dejar suficiente longitud para retirarla días después (figura 2).

Acto seguido, los hilos deben ser recuperados a través de cada puerto pélvico, uno de cada lado. Debido al riesgo de complicaciones intestinales, los hilos deben ser extraídos en un plano extraperitoneal. Para dicho efecto, se introduce por la incisión de la piel un instrumento "pescador" largo de acero, con la punta distal adaptada para recuperar los hilos. No se debe entrar a la cavidad sino buscar una disección extraperitoneal, superando el plano de los ovarios, hasta el punto donde se encuentran los hilos en el centro de la pelvis. El "pescador" rompe el peritoneo lo más cerca de los hilos y el ayudante toma uno de los hilos con la pinza de Maryland del otro puerto pélvico y lo introduce en el agujero presente en el extremo distal de este "pescador". Se extrae trayendo el hilo en el plano extraperitoneal hasta el orificio en la piel, donde se repara. Se repite el procedimiento en el otro lado (figura 3).

El retractor de Vecchietti es instalado en posición suprapúbica entre los dos puertos pélvicos. Enseguida, se montan los hilos en el mecanismo retractor de cada lado del aparato y se tensionan. Se dan las vueltas necesarias en ambos lados de manera que los hilos queden bien templados. El aparato queda sostenido en la pared abdominal solo por la tensión de los hilos a cada lado. La esfera ya no es visible (figura 4).

Se debe practicar media vuelta diaria a cada uno de los fijadores durante los siete días posoperatorios, lo cual equivale a un centímetro de longitud, aproximadamente. No es necesario agregar analgesia adicional. La sonda vesical se deja hasta el alta por el riesgo de retención urinaria que puede producir la presión de la esfera sobre la uretra y el trígono vesical.

Manejo posoperatorio: por protocolo se estableció la hospitalización posquirúrgica por siete días, durante los cuales se realizó tensión diaria del retractor y se enseñó a la paciente el uso del molde vaginal de silicona. En siete días se obtiene un canal vaginal entre 7 y $10 \mathrm{~cm}$, por expansión sostenida de la mucosa del introito. Puede dejarse algún tipo de marca en los hilos que sobresalen para calcular la profundidad que se va obteniendo. Al séptimo día se extrae la sonda vesical, se cortan los hilos desde el sistema retractor y se extrae la esfera por tracción de los hilos que salen por vagina. Luego se practica la introducción del molde de silicona hasta que la paciente sea capaz de hacerlo por sí misma, momento en el cual se da alta, con la instrucción de que lo use en forma permanente. Lo puede retirar para las funciones fisiológicas. El molde es lavable y para la introducción se prescribe un lubricante hidrofílico. Posteriormente, se cita a la paciente con 


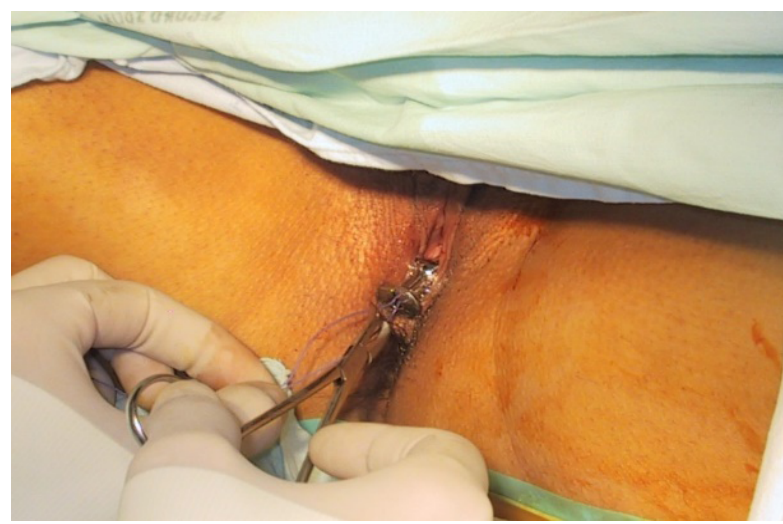

Figura 1. Paso de la aguja con mandril desde el introito hasta la cavidad abdominal

Fuente: cortesía del autor.

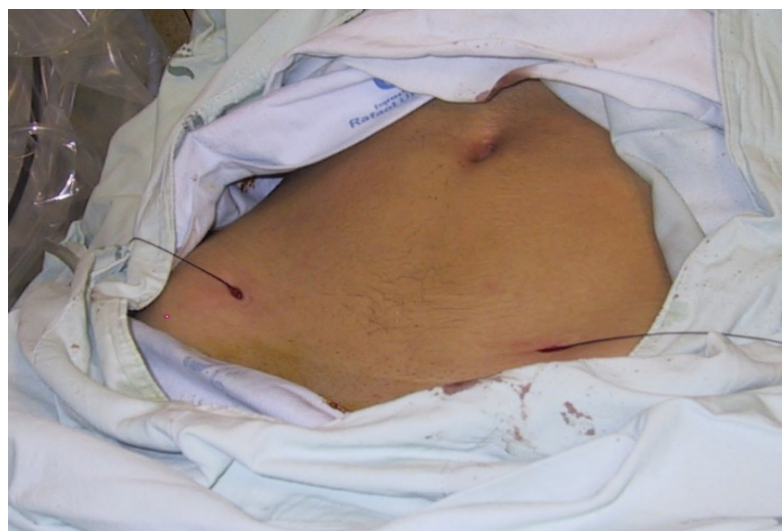

Figura 3. Esfera de poliuretano anudada a los hilos, en proceso de tracción hacia arriba

Fuente: cortesía del autor.

cortos intervalos de días las veces que sea necesario para asegurar el uso adecuado del molde. Se dan instrucciones para consultar en caso de dificultad para su introducción. El molde debe ser usado en forma continua por tres meses y luego en las noches durante un año. Actualmente se usan moldes cilíndricos rectos de silicona de $3 \mathrm{~cm}$ de diámetro.

Para el seguimiento se realizaron citas mensuales por tres meses y luego trimestrales hasta asegurar al menos un año de seguimiento. La longitud vaginal se definió al alta y trimestralmente hasta el primer año de seguimiento con la medición en centímetros completos de la penetración de la hoja inferior del espéculo en la neovagina.

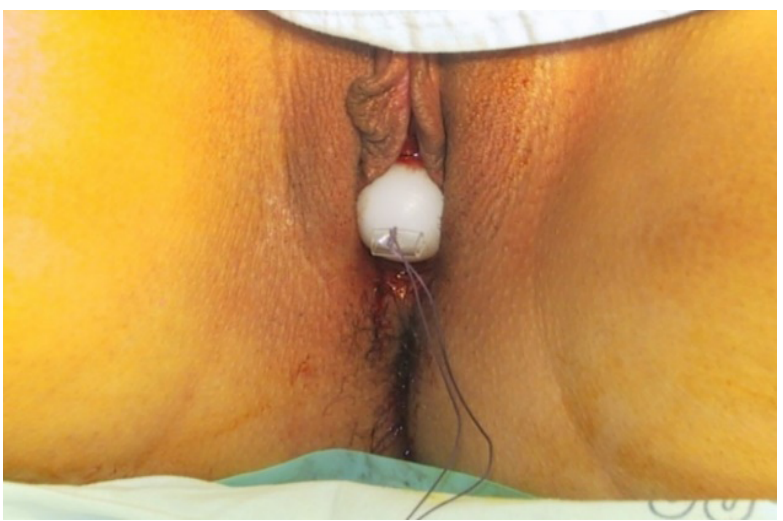

Figura 2. Hilos de polyglactin recuperados desde la pelvis por vía extraperitoneal

Fuente: cortesía del autor.

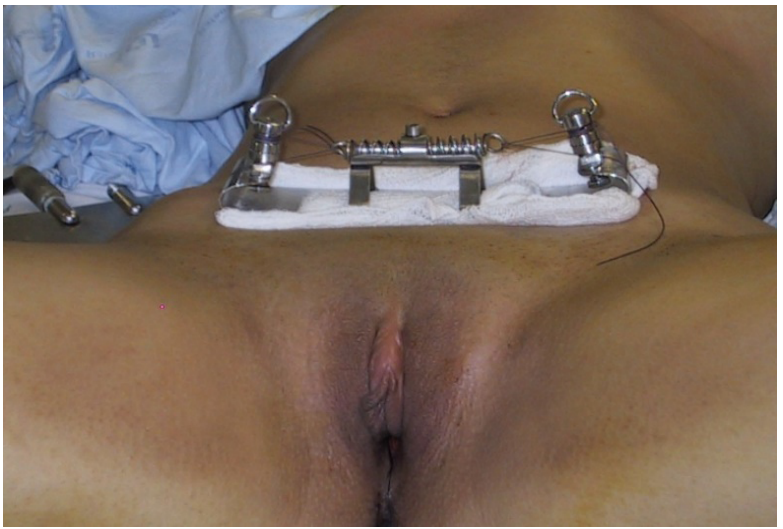

Figura 4. Aspecto final del retractor en el abdomen, con los hilos bajo tensión. La esfera ya no es visible

Fuente: cortesía del autor.

Variables medidas: edad, motivo de consulta inicial, diagnóstico de agenesia renal o malformaciones óseas en estudios de imágenes diagnósticas, duración del procedimiento en minutos a partir del registro anestésico, desde que se inicia el lavado quirúrgico hasta la terminación de las actividades de instrumentación quirúrgica. Como complicaciones intraoperatorias se buscaron la perforación vesical o el diagnóstico de hemorragia a partir de la cuantificación de la cantidad de sangre recuperada del frasco del aspirador y la impregnación de las compresas; como complicaciones posoperatorias, la infección y la estenosis de la neovagina. Como resultados de eficacia se evaluaron la presencia de 
relaciones sexuales y la satisfacción con la relación sexual coital.

Análisis estadístico. Se presentan valores absolutos y relativos, calculados con el total de mujeres intervenidas para las variables cualitativas, y medias con sus respectivos rangos para las variables cuantitativas. Estos cálculos se realizaron en el programa Excel de Microsoft Office.

Aspectos éticos. Las mujeres, o sus representantes legales según la edad, firmaron el consentimiento informado para el procedimiento. Se contó con la aprobación del comité de ética en investigación del Hospital Universitario San Vicente Fundación para hacer el estudio. Se garantizó la confidencialidad de la información y la privacidad de las pacientes.

\section{RESULTADOS}

En el periodo de estudio se atendieron 16 pacientes con agenesia vaginal, de las cuales 13 cumplieron con los criterios clínicos para la realización de TVV y todas se incluyeron en esta investigación. Tres pacientes fueron excluidas por lograr relaciones sexuales satisfactorias durante la espera de la cirugía. De las 13 pacientes incluidas, 11 casos tenían diagnóstico de MRKH y dos casos tenían diagnóstico de AIS. Se contó con seguimiento trimestral de 11 de ellas (84\%) durante al menos un año. Una paciente no regresó después del alta y la otra luego del tratamiento de hematómetra no asistió al seguimiento (figura 5).

La media de edad fue de 20,8 años (Rango: 1432). Los motivos de consulta fueron: en 9 (69,2\%) amenorrea primaria, en 3 (23\%) por apareunia y $1(7,7 \%)$ por dolor abdominal. Cuatro mujeres presentaban malformaciones asociadas: dos $(15,4 \%)$ tenían agenesia renal derecha, una (7,7\%) agenesia de peroné derecho, y una (7,7 \%) luxación congénita de cadera derecha. En tres casos $(23,0 \%)$ se les había practicado laparoscopia en otras instituciones.

Todos los procedimientos fueron laparoscópicos y el tiempo quirúrgico tuvo una media de 100 minutos (Rango: 60-180). La media del sangrado durante el procedimiento quirúrgico fue de 28,4 mL (Rango: 10-50). No hubo ninguna complicación asociada a la laparoscopia. En una paciente (7,7\%), se perforó la vejiga con la aguja. No hubo casos de paso rectal de la aguja. Las 13 pacientes obtuvieron una media de longitud vaginal al alta de 7,2 $\mathrm{cm}$ (Rango: 7-12). Con respecto a las complicaciones posoperatorias durante la observación, se presentaron se presentaron tres eventos $(23,1 \%)$. En un caso (7,7\%), la esfera rompió el tejido expandido y quedó

Figura 5.

Flujograma de pacientes incluidas en el de técnica de Vecchietti en el Hospital Universitario San Vicente Fundación de Medellín entre 2007 y 2012

Pacientes con agenesia vaginal

que consintieron participar en el estudio, $\mathrm{n}=16$

Total de pacientes incluidas en el estudio, $\mathrm{n}=13$

Lograron un canal vaginal durante el tiempo de preparación quirúrgica, $\mathrm{n}=3$ 
retenida por el peritoneo, luego se extrajo y no generó consecuencias negativas excepto por el dolor al momento de retirarla. Otra paciente presentó dehiscencia de la sutura de una de las incisiones pélvicas que se sostenía en el retractor. Se presentó una complicación tardía (7,7\%) relacionada con sangrado persistente por granuloma de contacto con el molde, que en ese tiempo era de acrílico, el cual requirió varios curetajes bajo anestesia. En un caso de una menor de edad, se presentó oclusión definitiva del espacio vaginal logrado debido al rechazo insuperable del uso del molde vaginal en el contexto del tratamiento urgente de un hematómetra.

Un total de 11 pacientes expresaron dificultades menores durante las relaciones sexuales en los primeros seis meses $(\mathrm{n}=11)$. $\mathrm{Al}$ año de seguimiento, 11 mujeres obtuvieron una profundidad vaginal media de 9,5 cm (Rango: 7-12) y fue posible introducirles el molde, sin molestias importantes. Las 11 pacientes manifestaron tener relaciones sexuales al año de seguimiento, de las cuales 10 (76,9\%) las consideraron satisfactorias. Una paciente (7,7\%) expresó la queja repetida de su compañero durante el coito, pero al examen permitía la introducción y apertura del espéculo desechable estándar de una manera fácil y amplia. Ninguna de las mujeres reportó problemas de lubricación. A las dos pacientes con AIS se les había realizado gonadectomía bilateral y recibían terapia hormonal con estrógenos orales (tabla 1).

\section{DISCUSIÓN}

Este estudio encontró que la TTV es segura, con baja frecuencia de complicaciones intra y posoperatorias, ninguna complicación seria, y eficaz en términos de funcionalidad y satisfacción de la paciente. Debido a que la única oclusión vaginal demostrada ocurrió en una paciente de 14 años, vale la pena considerar posponer este procedimiento, en lo posible, hasta cuando la mujer tenga necesidad de iniciar su vida sexual.

La técnica usada en nuestro servicio presenta variantes con respecto a la original. El paso de los hilos a través de una aguja desde el periné nos ha parecido más simple, cuando la comparamos con el paso desde la cavidad abdominal. Esta variante es descrita en otras publicaciones (13). La tensión diaria y la incomodidad del porte del retractor obligan a una hospitalización prolongada, la cual podría ser reducida con hospitalización en casa. No se hizo seguimiento de la calidad histológica del tejido vaginal obtenido, pero el aspecto al usar el espéculo no permitía diferenciarlo de una vagina normal. La respuesta sexual fue descrita como satisfactoria, lo cual no sorprende, pues esta depende en mayor medida del estímulo en el clítoris y las áreas vecinas.

En cuanto a la seguridad en nuestras pacientes no se presentaron las complicaciones publicadas en una revisión sistemática (14) que incluyó 162 estudios, que comparan las diferentes técnicas de neovagina, con 4326 pacientes entre los que se incluyen 939 casos de TVV. Los autores identificaron, en los casos tratados con TVV, 4 lesiones intestinales, 20 lesiones urológicas y 2 casos con hemorragia que requirieron transfusión de derivados sanguíneos. En cuanto a complicaciones posoperatorias inmediatas informan 24 infecciones, 5 hematomas y 2 necrosis de la cúpula vaginal. A largo plazo, informan 5 estenosis y 3 casos de granuloma, complicaciones también presentes en nuestro estudio. Cetin, en Turkia, en 62 casos tampoco informa complicaciones serías con esta técnica (13).

Respecto a la funcionalidad, nuestros resultados son similares a los informados por Pastor et al. en 42 casos intervenidos con la TVV, en los que informan una adecuada función sexual según el Índice de función sexual femenina (15).

\section{CONCLUSIONES}

La TVV es un procedimiento seguro, que permite obtener vaginas funcionales. Se requieren estudios con grupo control para tener una mejor evaluación de la eficacia de las diferentes técnicas de construcción de la neovagina. 
Tabla 1.

Resumen de resultados de las pacientes con agenesia vaginal a quienes se les practicó vaginoplastia de Vecchietti en el Hospital San Vicente Fundación, en Medellín, Colombia, 2007 y 2012

Antecedente personal de otras malformaciones

\section{Ninguna \\ Agenesia renal derecha \\ Agenesia de peroné derecho \\ Luxación congénita cadera derecha}

\begin{tabular}{|c|c|}
\hline Antecedente personal de otras malformaciones & $\begin{array}{l}\text { Número de pacientes, } \\
\qquad n=13(\%)\end{array}$ \\
\hline Ninguna & $9(69,2)$ \\
\hline Agenesia renal derecha & $2(15,4)$ \\
\hline Agenesia de peroné derecho & $1(7,7)$ \\
\hline Luxación congénita cadera derecha & $1(7,7)$ \\
\hline \multicolumn{2}{|l|}{ Motivo de consulta } \\
\hline Amenorrea primaria & $9(69,2)$ \\
\hline Dolor abdominal & $1(7,7)$ \\
\hline Apareunia & $3(23)$ \\
\hline \multicolumn{2}{|l|}{ Ayuda diagnóstica } \\
\hline Resonancia (RMN) & $5(38,4)$ \\
\hline Ecografía & $3(23,0)$ \\
\hline RMN + ecografía & $2(15,4)$ \\
\hline Laparoscopia + ecografía & $1(7,7)$ \\
\hline Laparoscopia + RMN & $1(7,7)$ \\
\hline Laparoscopia + ecografía + RMN & $1(7,7)$ \\
\hline \multicolumn{2}{|l|}{ Complicaciones } \\
\hline No presentaron & $9(69,2)$ \\
\hline \multicolumn{2}{|l|}{ - Tempranas } \\
\hline Lesión vesical accidental (transoperatorio) & $1(7,7)$ \\
\hline Desgarro vaginal (posoperatorio) & $1(7,7)$ \\
\hline Dehiscencia de sutura (posoperatorio) & $1(7,7)$ \\
\hline \multicolumn{2}{|l|}{ - Tardías } \\
\hline Estrechamiento del canal postratamiento de hematómetra (seguimiento) & $1(7,7)$ \\
\hline \multicolumn{2}{|c|}{ Longitud final al año de seguimiento } \\
\hline $12 \mathrm{~cm}$ & $2(15,4)$ \\
\hline $10 \mathrm{~cm}$ & $5(38,4)$ \\
\hline $8,0 \mathrm{~cm}$ & $3(23,0)$ \\
\hline $7,0 \mathrm{~cm}$ & $1(7,7)$ \\
\hline Desconocida & $2(7,7)$ \\
\hline \multicolumn{2}{|l|}{ Satisfacción sexual } \\
\hline Satisfecha & $10(76,9)$ \\
\hline Insatisfecha & $1(7,7)$ \\
\hline Desconocida (no asistieron a controles) & $2(15,4)$ \\
\hline
\end{tabular}

Número de pacientes, $\mathbf{n}=13(\%)$

$9(69,2)$

$2(15,4)$

$1(7,7)$

$1(7,7)$

$9(69,2)$

Antecedente personal de otras malformaciones 


\section{REFERENCIAS}

1. Capraro VJ, Gallego MB (1976) Vaginal agenesis. Am J Obstet Gynecol. 1976;124(1):98-107. DOI: https:// doi.org/10.1016/0002-9378(76)90020-X

2. Michala L, Cutner A, Creighton SM. Surgical approaches to treating vaginal agenesis. BJOG. 2007;114(12):1455-9. https://doi.org/10.1111/ j.1471-0528.2007.01547.x

3. Creatsas G, Deligeoroglou E. Vaginal aplasia and reconstruction. Best Pract Res Clin Obstet Gynaecol. 2010;24(2):185-91. https://doi.org/10.1016/j. bpobgyn.2009.11.002

4. Bouman M, van Zeijl MC, Buncamper ME, et al. Intestinal vaginoplasty revisited: A review of surgical techniques, complications, and sexual function. J Sex Med. 2014;11: 1835-47. https://doi.org/10.1111/ jsm. 12538

5. Pravin M, Jyoti M, Rakhi S. New laparoscopic peritoneal pull-through vaginoplasty technique. Reprod Sci. 2014;24(6):181-6.

6. Edmons DK, Rose GL, Lipton MG, Quek J. MayerRokitansky-Küster-Hauser Syndrome: A review of 245 consecutive cases managed by a multidisciplinary approach with vaginal dilators. Fertil Steril. 2012;97(3):686-90. https://doi.org/10.1016/j.fertnstert.2011.12.038

7. ACOG Committee on Adolescent Health Care. ACOG Committee Opinion. Nonsurgical diagnosis and management of vaginal agenesis. Obstet Gynecol. 2002;100(1):213-6. https://doi. org/10.1097/00006250-200207000-00034

8. Kuhn A, Neukomm C, Dreher EF, Imobersteg J, Mueller MD. Prolapse and sexual function 8 years after neovagina according to Shears: A study of 43 cases with Mayer-von Rokitansky-Küster-Hauser syndrome. Int Urogynecol J. 2013;24(6): 1047-52. https://doi. org/10.1007/s00192-012-1980-z
9. Templeman CL, Lam AM, Hertweek SP. Surgical management of vaginal agenesis. Obstet Gynecol Surv. 1999;54(9):583-91. https://doi. org/10.1097/00006254-199909000-00023

10. Vecchietti G. Le neo-vagin dans le syndrome de Rokitansky-Küster-Hauser. Rev Med Suisse Romande. 1979;99(9):593-601.

11. Brucker SY, Gegusch M, Zubke W, Rall K, Gauwerky JF, Wallwiener D. Neovagina creation in vaginal agenesis: Development of a new laparoscopic Vecchietti-based procedure and optimized instruments in a prospective comparative interventional study in 101 patients. Fertil Steril. 2008;90(5):1940-52. https:// doi.org/10.1016/j.fertnstert.2007.08.070

12. Fedele L, Busacca M, Candiani M, Vignali M. Laparoscopic creation of a neovagina in Mayer-RokitanskyKüster syndrome by modification of Vecchietti's operation. Am J Obstet Gynecol. 1994;171(1):268-9. https://doi.org/10.1016/0002-9378(94)90482-0

13. Cihan C, Cenk S, Ibrahim F, Murat A, Turan C. Long-term outcomes of laparoscopic bean vaginoplasty (modified Vecchietti procedure) for MayerRokitansky-Kuster-Hauser Syndrome. J Laparoendosc Adv Surg Tech. 2016;26(8): 591-5. DOI: https://doi. org/10.1089/lap.2016.0031

14. McQuillan SK, Grover SR. Dilation and surgical management in vaginal agenesis: A systematic review. Int Urogynecol J. 2014;(25):299-311. https://doi. org/10.1007/s00192-013-2221-9

15. Pastor Z, Froněk J, Nováčková M, Chmel R. Sexual life of women with Mayer-Rokitansky-Küster-Hauser Syndrome after laparoscopic Vecchietti vaginoplasty. Sex Med. 2017;5(2):e106-e113. https://doi. org/10.1016/j.esxm.2016.12.003 